\title{
UNDERSTANDING THE FRACTIONATION OF OILS VIA NATURAL AND INDUCED MIGRATION FROM UNCONVENTIONAL RESERVOIRS
}

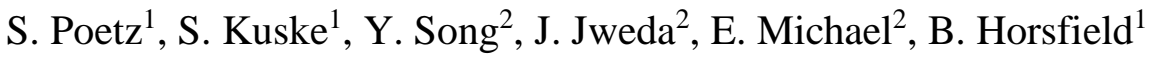 \\ ${ }^{1}$ Helmholtz Centre Potsdam GFZ German Research Centre for Geosciences, Germany \\ ${ }^{2}$ ConocoPhillips, United States of America
}

\section{Introduction}

Understanding the fate of organic matter within the source-carrier-reservoir system under geological PT-conditions, we need to predict the physical properties of the generated, retained, and migrating petroleum fluids. The physical properties of these bulk fluids are defined by the chemical composition of component fractions, which in turn strongly depends on the chemical composition of the mature source kerogen and fractionation processes that have occurred during expulsion, migration, and production. The migration behaviour of petroleum fluids and their organic constituents is one of the key factors in petroleum systems. In conventional systems, fluids migrate naturally from a source to a reservoir located within a distance of few metres to hundreds of kilometres. Migration fractionation typically leads to hydrocarbon-enriched migrated fluids and in-source retained fluid typically enhanced with polar organic compounds containing Nitrogen, Sulfur or Oxygen (NSO compounds) (Brenneman and Smith 1958). In unconventional systems, hydrocarbons are produced directly from the in-situ organic matter in the source rock using hydraulic stimulation techniques. Retained fluids undergo 'induced migration' via production through fracture networks created by high-pressure injection of 'fracking fluids'.

While the basic mechanisms of the fractionation processes in conventional petroleum systems are established, much less is known about production fractionation in unconventional reservoirs (induced migration). Moreover, our knowledge about NSO compound partition behaviour is essentially restricted to relatively low molecular weight compounds (Larter et al. 1996). A more detailed characterization of the medium to large molecular weight petroleum NSO fraction is afforded by ultrahigh resolution mass spectrometry (Fourier transform ion cyclotron resonance mass spectrometry, FT-ICR MS) used with different ionization modes (Electrospray ionization, ESI, and Atmospheric pressure photoionization, APPI (e.g. Mahlstedt et al. 2016).

In this study, we investigate migration fractionation caused by natural and induced migration within the Eagle Ford formation in Texas (USA). We have conducted a comprehensive comparison of retained and migrated fluids' compositional analyses using source rock samples, conventionally produced black oils from the Austin Chalk and tight oils produced from the Eagle Ford formations. The impact of migration processes on bulk properties and different compound classes like light hydrocarbons, $n$-alkanes, biomarkers, aromatic hydrocarbons and NSO compounds as well as on carbon isotopic composition of $n$-alkanes is assessed in detail.

\section{Results}

Eagle Ford tight oils show an overall strong enrichment in $n$-alkanes, especially of those with up to ten carbon atoms. Its aromatic and cycloalkane fractions are strongly decreased as compared to the source rock bitumen. With respect to NSO compounds, we observed a strong 


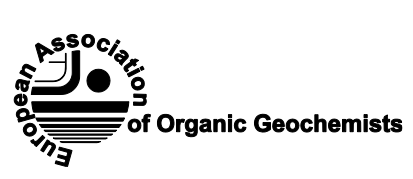

overall depletion in compounds of higher polarity (e.g acidic compounds), while medium polarity NSO compounds display compositional changes. Namely, compounds with oxygen (e.g. phenols, ketones) and sulphur and oxygen (e.g. sulphoxides) seem to be retained within the source as well as highly aromatic (high number of double bond equivalents) and high polarity (less alkylated) NSO compounds. In contrast, compounds with nitrogen and oxygen are extremely enriched during induced migration. The high fractions of sodiated ions in tight Eagle Ford produced oils might reflect the water chemistry of the fracking fluids. The carbon isotopic composition of light hydrocarbons in Eagle Ford tight oils and conventional Austin Chalk black oils differ while that of medium and long chain $n$-alkanes does not.

\section{Conclusions}

This is the first time fractionation in tight oils produced via hydraulic stimulation is described on a molecular level and compared to naturally migrated fluids, using the example of the Eagle Ford Formation. Tight oil production is accompanied by fractionation with respect to molecular size, molecular shape and functional groups expressing differences in physical properties like volatility, volume, adsorption potential on kerogen or mineral surfaces or solubility in the water based fracking fluids.
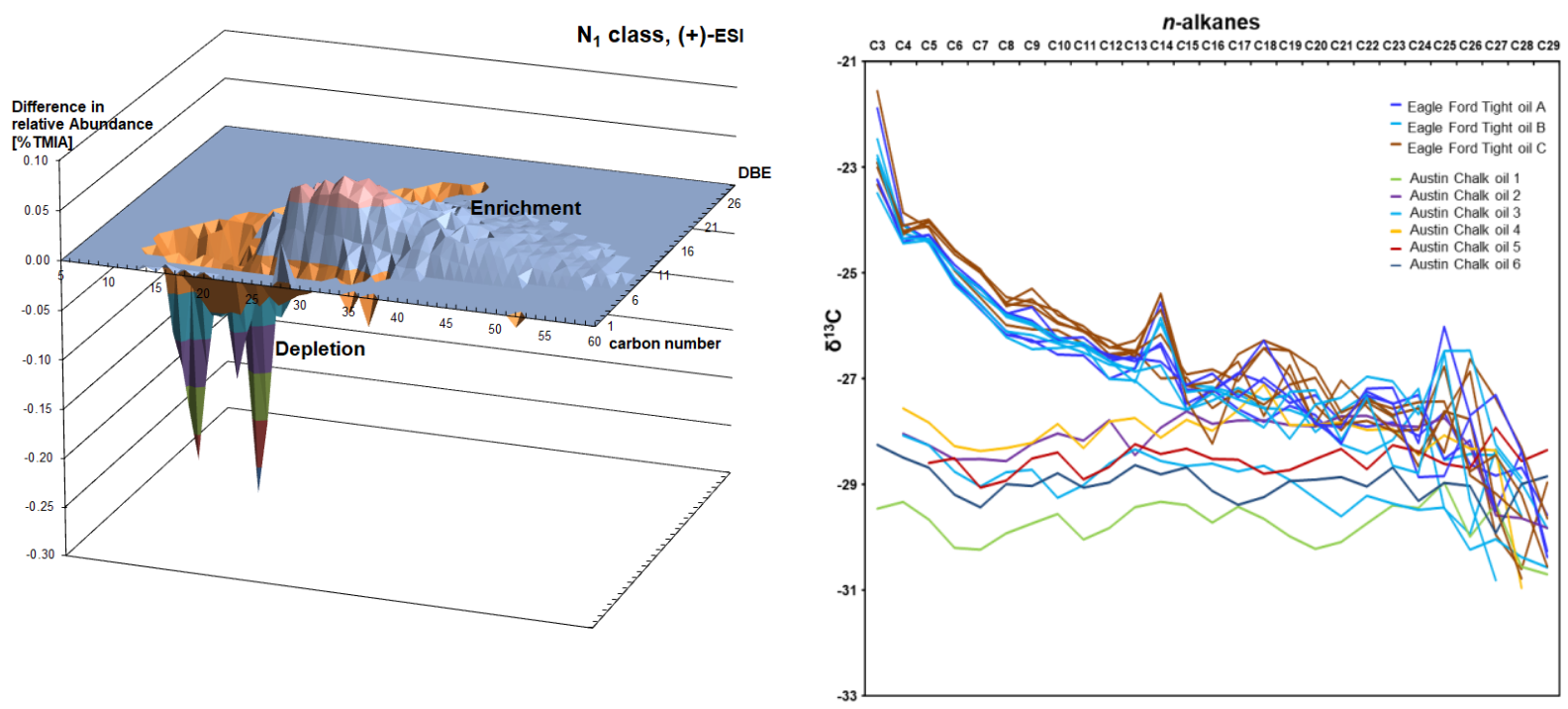

Figure 1 Left: Enrichment and depletion of basic $N_{1}$ compounds via induced migration. Right: Differences in compound specific carbon isotopic composition of Eagle Ford tight oils and Austin Chalk reservoired oils.

\section{References}

Brenneman, M. C.; Smith, P. V., Jr. The chemical relationships between crude oils and their source rocks. In Habitat of oil: A symposium; Weeks, L. G., Ed.; American Association of Petroleum Geologists: Tulsa, OK, 1958; pp 818-849.

Larter SR, Bowler BF, Horstad I. Molecular indicators of secondary oil migration distances. Nature (1996). 383 (6601), 593-597.

Mahlstedt N, Horsfield B, Wilkes H, Poetz S. Tracing the impact of fluid retention on bulk petroleum properties using nitrogen-containing compounds. Energy \& Fuels (2016). 30, 6290-6305. 